\title{
Influence of sacrificial bonds on the mechanical behaviour of polymer chains
}

\section{S. Soran Nabavi}

Institute of Physics, Montanuniversitaet Leoben, Leoben, Austria

2 Matthew J. Harrington

Department of Biomaterials, Max-Planck-Institute of Colloids and Interfaces, Potsdam, Germany
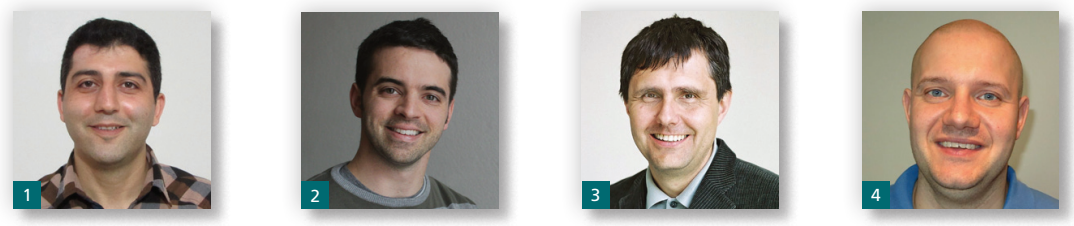

A growing focus in modern materials science is the attempt to transfer principles found in nature into new technological concepts with the goal of producing novel materials with tailored mechanical properties. One of these principles used in nature is the concept of sacrificial bonding (i.e. non-covalent cross-links that rupture prior to the protein backbone), which is associated with increased toughness in many biological materials. In the present work, the influence of the number and distribution of sacrificial bonds (SBs) on three main mechanical parameters-strength, work to fracture and apparent stiffness-is investigated in a simple model system using computer simulations. The results show that the work to fracture is mainly determined by the number of SBs present in the system, while the apparent stiffness and, to a lesser extent, the strength is altered when the distribution of SBs is changed.

\section{Introduction}

In contrast to engineered materials, biological organisms utilize a relatively limited selection of building blocks to synthesize materials (e.g. proteins, sugars, environmentally abundant ions). In spite of this, however, natural materials span an extremely wide range of mechanical properties, which is achieved by hierarchical structuring of the material over multiple length scales and by a combination of materials with opposing mechanical properties. ${ }^{1}$ One common and successful strategy to increase the toughness of protein-based biological materials is to use so-called sacrificial bonds $(\mathrm{SBs}){ }^{2}$ These non-covalent cross-links are weaker than the covalent bonds that comprise the protein backbone, and consequently, upon loading, they rupture before the covalent bonds fail. By doing so, SBs reveal hidden length (i.e. the length increase associated with unfolding of folded proteins) providing an efficient energy dissipation mechanism, while the overall material integrity survives. ${ }^{3}$ Furthermore, these bonds are reversible and may reform when the load is released, allowing for molecular repair. SBs have been found in a large variety of biologial materials like wood, ${ }^{4}$ bone $^{5-7}$ and in some softer biological fibres such as silk, ${ }^{8}$ whelk egg capsule $^{9}$ and mussel byssus threads. ${ }^{10-13}$
In materials such as silk, SBs are often weak hydrogen bonds combined in large numbers in regular protein conformations in order to collectively produce high stiffness ${ }^{14}$; however, in the case of the mussel byssus, much stronger interactions between metal ions and proteins are employed. In this regard, the mussel byssus is an especially fascinating material. The mussel secretes the collageneous byssal threads as a means of creating a secure attachment in wave-swept rocky seashore habitats. Among the impressive properties of the mussel byssus are its high extensibility of over $100 \%$, high stiffness and toughness, ${ }^{10}$ its hard and wearresistant outer coating ${ }^{15-18}$ and, last but not least, its ability to create strong and long-lasting underwater adhesion to a variety of surfaces. ${ }^{17} \mathrm{~A}$ fundamental aspect shaping each of these properties is the interaction of proteins with metal ions. In particular, histidinezinc and 3,4-dihydroxyphenylalanine (DOPA)-iron coordination complexes in the protein building blocks of the material serve as reversible SBs with a relatively high binding energy of 20-30\% of a covalent bond. ${ }^{19,20}$

Recently, a number of technological materials have emerged that attempt to draw inspiration from the aforementioned notable 
Bioinspired, Biomimetic and Nanobiomaterials Volume 3 Issue BBN3
Influence of sacrificial bonds on the mechanical behaviour of polymer chains Nabavi, Harrington, Fratzl and Hartmann properties of the mussel byssus, for example, strong biocompatible surgical adhesives, ${ }^{21}$ implantable drug-eluting devices in human blood vessels capable of withstanding blood flow ${ }^{22}$ and super tough hydrogel materials that may serve as artificial cartilage. ${ }^{23}$ Additionally, a new generation of biomimetic self-repairing metallopolymers has arisen, which directly draw inspiration from the byssal threads. ${ }^{24-27}$ In spite of these advances, many challenges still persist. Thus, a thorough experimental and theoretical understanding of the molecular processes underlying the SB mechanism is crucial.

In particular, the specific details of the topology and nature of the metal-binding domains have been found to be increasingly important in determining material properties. It was shown, for example, that the type of coordination bond (i.e. the metal and ligand utilized) affects the stiffness and strength of a polymer ${ }^{25,27,28}$ and that the fracture toughness as well as the extensibility of materials based on SBs is strongly dependent on the pulling speed. ${ }^{29,30}$ Furthermore, it was shown that the topology of SBs and thermal backbone fluctuations strongly affect the efficacy of $\mathrm{SBs}^{31}$ and that the spatial distribution of SBs can have a strong effect on mechanics ${ }^{32}$ - a random distribution of SBs is necessary to provide the system with shear deformability. Along these lines, the histidine-rich domains in the load-bearing proteins comprising the self-healing mussel thread core contain numerous variations in sequence depending on the protein variant and species. ${ }^{33}$ Sequence variations result in differences in distribution and number of metal-binding histidine residues and are believed to be associated with differences in the mechanics between threads from different species. ${ }^{33}$ It has been demonstrated that the binding energy of histidine-metal complexes can be greatly affected by the number and spacing of histidine residues in short peptides ${ }^{34}$ and that the position of the metal-coordination bond in the folded protein chain influences the mechanical stability of the proteins. ${ }^{35}$ In the current study, the influence of the distribution of SBs on the mechanical properties in fibrous systems inspired by the mussel byssus is further investigated utilizing computer simulations. Monte Carlo simulations mimicking tensile experiments were carried out on a simple model system, and the main mechanical properties (strength, work to fracture and apparent stiffness) as a function of the number and distribution of SBs was assessed. It was shown that the work to fracture is mainly determined by the number of SBs, while their distribution has a significant impact on the strength and apparent stiffness.

\section{The model}

The histidine-rich regions of the load-bearing proteins in the tough fibrous core of mussel byssal threads ${ }^{10,33}$ and the DOPA-rich proteins found in the plaque ${ }^{12}$ and the protective cuticle ${ }^{18}$ form protein-metal SBs that provide the inspiration for the presented model, already introduced in Ref. 31. The histidine-rich sequences from the byssal core, for example, are relatively short segments that consist typically of 30-80 amino acids. ${ }^{33}$ This motif is described by a linear chain of $N=50$ covalently bonded beads. To prevent self-interaction of the chain, the beads were assigned a hard-sphere radius $R$ that essentially forbids the overlap of two beads and that we set as the unit of length in the simulations. The covalent bonds were described via a Morse potential

$$
\text { 1. } E\left(r_{i j}\right)=E_{0}\left[\left\{1-\exp \left[-\beta\left(r_{i j}-r_{0}\right)\right]\right\}^{2}-1\right]
$$

$E_{0}=5 \mathrm{eV}$ is the depth of the potential, $\beta^{-1}=0.5 R$ is the width of the potential, $r_{0}=3 R$ is the equilibrium distance and $r_{i j}$ is the distance between two neighbouring beads (Figure 1b). Consequently, the contour length of the chain is given by $L_{c}=(N-1) r_{0}$. SBs were described by defining $N_{\mathrm{s}}$ of the beads as sticky, setting the sticky site density as $\rho_{\mathrm{s}}=N_{\mathrm{s}} / N$. SBs in this model always formed from only two sticky sites and were allowed to open and close reversibly (see Figure 1a for a schematic sketch of the model). Not much is known

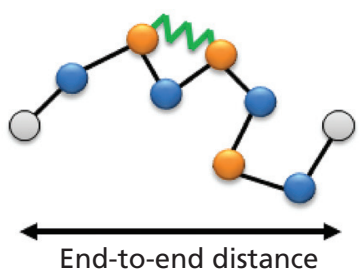

(a)

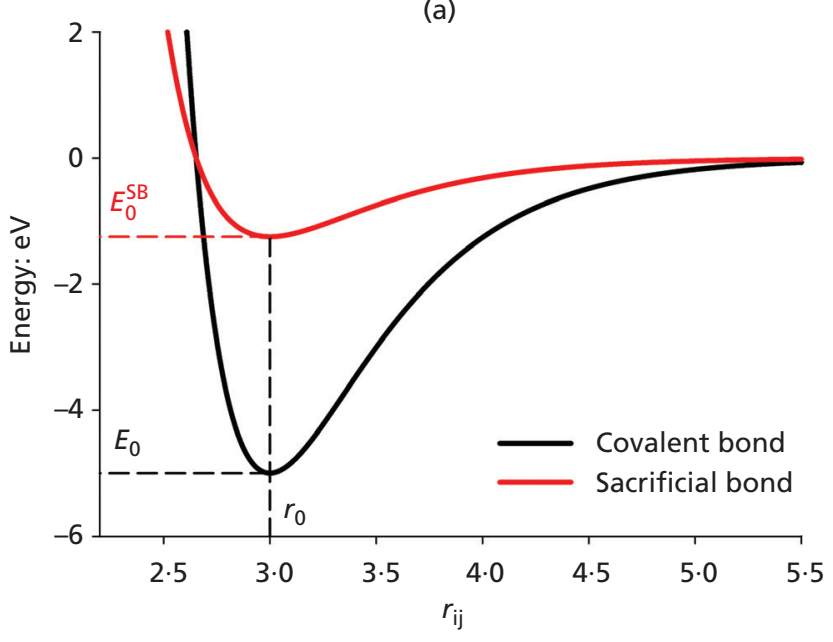

(b)

Figure 1. (a) Sketch of the used model. Gray beads denote the two fixed outer beads defining the end-to-end distance, non-sticky sites are shown in blue and sticky sites in orange, respectively. Covalent bonds are indicated by black bars, while a closed sacrificial bond is shown by the green zigzag line. (b) The Morse potential used to describe the energetics of covalent (black) and sacrificial (red) bonds. The vertical dotted line indicates the equilibrium distance $r_{0}$, while the two horizontal lines indicate the used binding energies $E_{0}$ and $E_{0}^{\mathrm{SB}}$, respectively. 
Bioinspired, Biomimetic and Nanobiomaterials Volume 3 Issue BBN3
Influence of sacrificial bonds on the mechanical behaviour of polymer chains Nabavi, Harrington, Fratzl and Hartmann about the energetics of SBs. Nevertheless, there are experimental indications that the binding energy of a histidine-metal complex lies between 20 and $30 \%$ of a covalent bond. ${ }^{19,20}$ For the sake of simplicity, in this study, the SBs are also described with a Morse potential as are the covalent bonds. The binding energy of the SBs is set to $25 \%$ of the value of a covalent bond, $E_{0}^{\mathrm{SB}}=1 \cdot 25 \mathrm{eV}$.

In the simulations, we investigated two different sticky site densities ( $\rho_{\mathrm{s}}=0.08$ and $0 \cdot 24$, respectively) and three different arrangements of sticky sites: $(a)$ ordered, $(b)$ patches and $(c)$ random (see top row of Figure 3). In the ordered case, the sticky sites were introduced regularly with always the same number of non-sticky sites in between. In the second arrangement, the chain is divided into three segments of equal length (the patches). The sticky sites are distributed regularly on the outer two patches, while the middle patch is sticky site free. In the third arrangement, the sticky sites are distributed randomly under the additional constraint that two sticky sites are not allowed to be directly neighbouring.

In the simulations, load-displacement curves were obtained using the Helmholtz ensemble. ${ }^{36}$ The end-to-end distance L of the polymer was defined by pinning its two outer beads. The position of the inner beads and the SBs were updated using a standard Metropolis algorithm ${ }^{37}$ and the force on the outer beads was recorded and averaged. Starting from a small end-to-end distance $L$ was slowly increased until $L / L_{\mathrm{C}}=1$. Up to 20 independent simulation runs have been done for each density and arrangement. Up to 3 million Monte Carlo steps (i.e. jump trials per bead) were performed for each single point. The temperature was set to the ambient value of $k_{B} T=25 \mathrm{meV}$.

\section{Results and discussion}

Figure 2 shows the averaged load-displacement curves of a single polymer chain with $\rho_{\mathrm{s}}=0.08$ for an ordered and random arrangement of sticky sites. In the ordered arrangement, five peaks can be observed. The first four peaks correspond to the rupture of SBs with different topologies, the last peak is due to backbone stretching (Figure 2a). ${ }^{31}$ Each of these single peaks can be well fitted with the worm-like chain model $^{38}$ that was shown to describe entropic forces due to the tendency of elongated polymers to recoil. ${ }^{39,40}$ A parameter that is connected to the toughness of the material is the work to fracture $W_{0}=W+\Delta W$ that is given by the area under the curve. $W=11.38 \mathrm{eV}$ is the work to straighten the molecule, that is, to elongate the molecule to its contour length, and $\Delta W=25 \mathrm{eV}$ is the additional contribution due to the rupture of the covalent backbone. Because all SBs are open when the chain is fully elongated, $\Delta W$ is the same for all configurations and is omitted in the following. The strength of the material $F_{\mathrm{m}}$ due to SBs is calculated as the maximum load of the curve after subtracting the pure, covalent interaction. For the ordered distribution of sticky sites, the rather high value of $F_{\mathrm{m}}=0.77 \mathrm{eV} / \mathrm{R}$ is found. Nevertheless, due to thermal fluctuations of the backbone, this

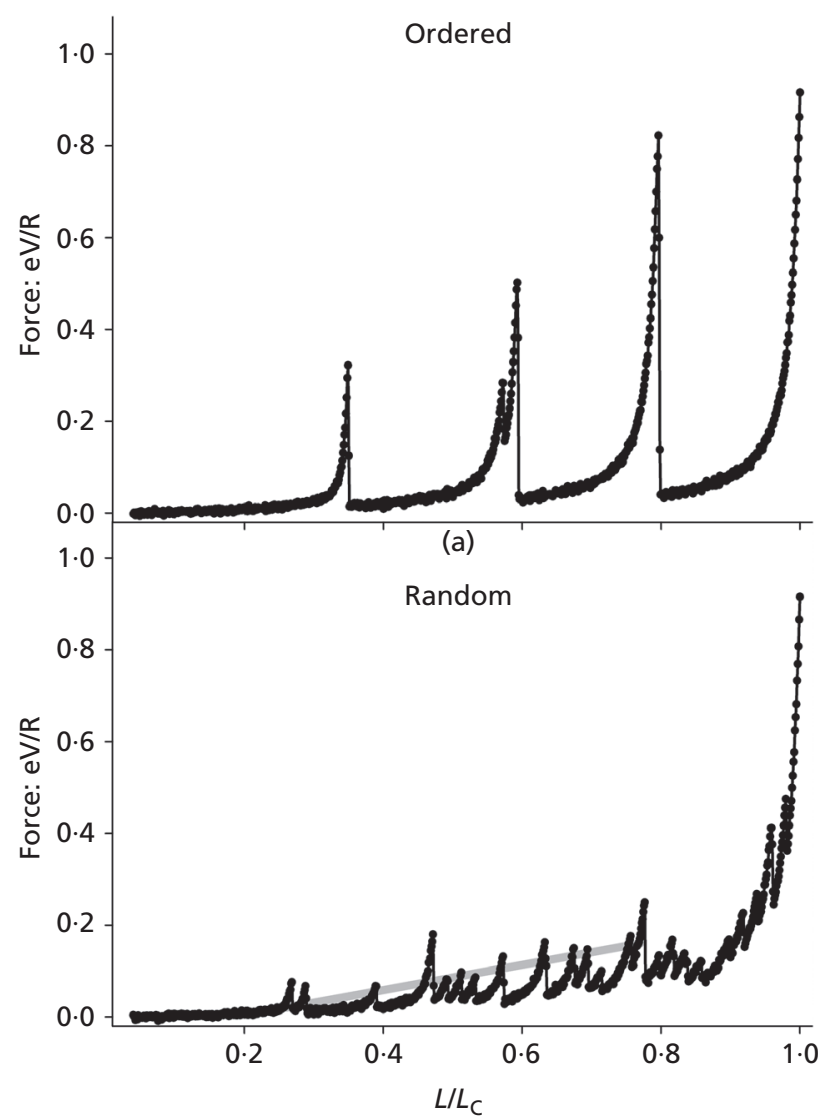

(b)

Figure 2. Load-displacement curves for an (a) ordered and (b) random arrangement of sticky sites with $N=50, k_{B} T=25 \mathrm{meV}$ and $\rho_{\mathrm{s}}=0.08$. In the random arrangement, the apparent stiffness of the material (i.e. the mean slope of the initial part of the curve) is indicated.

value is considerably lower than the theoretical strength of one SB $F_{m}^{\text {th }}=E_{0} \beta / 2=1 \cdot 25 \mathrm{eV} / \mathrm{R}^{31}$

In the random distribution of sticky sites, 20 independent configurations were generated and tested. The single force peaks of the loading curve are smeared out compared to the ordered distribution, an effect that considerably reduces the strength to $F_{\mathrm{m}}=0 \cdot 26 \mathrm{eV} / \mathrm{R}$. Because of the randomness of the sticky site distribution, their positions along the chain and thus the corresponding positions of the force peaks are not fixed. Consequently, the load is distributed over the entire range of the load-displacement curve leading to a lowering of the peak height compared to the ordered case. Nevertheless, within error, the work to fracture $W=11.45 \mathrm{eV}$ is identical with the result for the ordered arrangement. Furthermore, the random arrangement allows one to define an apparent stiffness $E$ of the material that is defined as the mean slope of the first increase in the load-displacement curve. It is found that $E=1.84 \mathrm{meV} / \mathrm{R}^{2}$. Analogous to an ordinary stiffness, the apparent stiffness is a measure of how much force is needed 


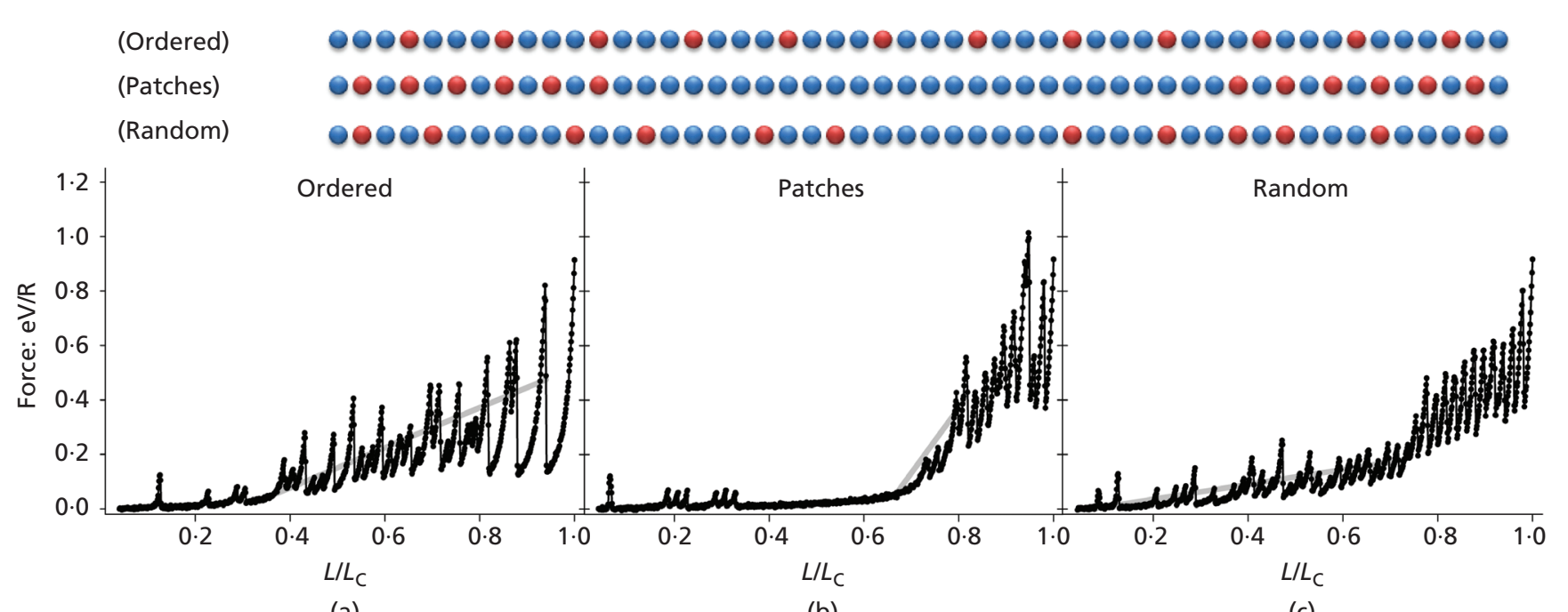

(a)

Figure 3. Load-displacement curves for three different distributions of sticky sites ((a) ordered, (b) patches and (c) random) and $N=50, k_{B} T$ $=25 \mathrm{meV}$ and $\rho_{\mathrm{s}}=0.24$. The gray lines indicate the apparent stiffness of the materials defined as the mean slope of the first part of the (b)

(c)

load-displacement curve. In the top part of the figure, a sketch of the different arrangements of sticky sites are shown (sticky sites are shown in red, non-sticky sites in blue). to elongate the structure to a certain length. While the ordinary stiffness describes the elastic behaviour of a material, the apparent stiffness is determined by the rupture of bonds that is characteristic for plastic deformation. Nevertheless, due to the reversibility of the SBs, they are able to reform over time following unloading, and the initial apparent stiffness is recovered.

Figure 3 shows the load-displacement curves for a high sticky site density $\rho_{\mathrm{s}}=0.24$ and three different arrangements of sticky sites. In the ordered arrangement, two successive sticky sites are separated by three non-sticky sites. In contrast to the low-density ordered arrangement, the stretching part of the load-displacement curve does not show single peaks corresponding to SB rupture, but rather a continuous shape (Figure 3a). This shows that after a single SB rupture, the force does not drop to zero but is taken up by some other SBs. Similar to the low-density arrangement (Figure 2), this allows the definition of an apparent stiffness $E=$ $5.03 \mathrm{meV} / \mathrm{R}^{2}$ that is also indicated by the gray line in the figure. The strength of this material is found to be $F_{\mathrm{m}}=0.7 \mathrm{eV} / \mathrm{R}$ and the work to fracture $W=22.55 \mathrm{eV}$.

The load-displacement curve changes significantly when the arrangement of sticky sites is changed to the patches configuration (Figure $3 \mathrm{~b}$ ). When stretched, the intermediate patch that contains non-sticky sites only fully elongates before the SBs starts to rupture. Thus, the force starts rising only when the chain is stretched to $L / L_{\mathrm{C}}=0.7$ (the few rupture events at small end-to-end distances correspond to SBs that have been formed between sticky sites in different patches). Compared to the ordered distribution, this late onset of the force slightly reduces the area under the curve to $W=$
$19.63 \mathrm{eV}$, but largely enhances the apparent stiffness of the material to $E=16 \cdot 26 \mathrm{meV} / \mathrm{R}^{2}$. The reason for this large value of the apparent stiffness is that due to the late onset of the force, the force has to rise over a smaller distance, yielding a large slope. The strength of the material is given by $F_{\mathrm{m}}=0.9 \mathrm{eV} / \mathrm{R}$.

Finally, Figure $3 \mathrm{c}$ shows the load-displacement curve for the random arrangement and $\rho_{\mathrm{s}}=0 \cdot 24$. The curve shows two distinct regions: below $L / L_{\mathrm{C}}=0 \cdot 8$, an irregular region of low force and above this value, a more regular region of higher forces. The first of these two regions corresponds to the rupture of SBs that connect sticky sites that are far apart along the chain. Due to their long distance along the chain, these SBs rupture at smaller end-to-end distances than SBs between neighbouring sticky sites. Nevertheless, in this regime, the number of intact SBs stays roughly constant showing that new SBs are reformed, but now, between sticky sites in closer distances along the chain. When the elongation $L / L_{\mathrm{C}}=0.8$ is reached, the chain is stretched to the extent that $\mathrm{SB}$ reformation ceases, and on further elongation, the number of intact SBs rapidly decreases to zero. It is the large number of SB rupture events distributed over a short length that is responsible for the higher value of the force in the region above $L / L_{\mathrm{C}}=0 \cdot 8$. Due to the small number of sticky sites for the random arrangement and $\rho_{\mathrm{s}}=0.08, \mathrm{SB}$ reforming is not possible, and the load-displacement curve shows a single region of low force only (Figure 2b). The random arrangement with $\rho_{\mathrm{s}}=0 \cdot 24$ shows an apparent stiffness of $E=1.84 \mathrm{meV} / \mathrm{R}^{2}$, a work to fracture $\mathrm{W}=23.51 \mathrm{eV}$ and a strength of $F_{\mathrm{m}}=0.55 \mathrm{eV} / \mathrm{R}$.

Table 1 summarizes the different mechanical parameters obtained for the different structures characterized by the number 


\begin{tabular}{ccccc}
\hline & & Work to fracture $(\boldsymbol{W}): \mathbf{e V}$ & Apparent stiffness $(\boldsymbol{E}): \mathbf{m e V} / \mathbf{R}^{2}$ & Strength $(\boldsymbol{F m}): \mathbf{e V} / \mathbf{R}$ \\
\hline$\rho_{\mathrm{s}}=0.08$ & Random & 11.45 & 1.84 & 0.26 \\
& Ordered & 11.38 & - & 0.77 \\
$\rho_{\mathrm{s}}=0.24$ & Random & 23.51 & 1.84 & 0.55 \\
& Ordered & 22.55 & 5.03 & 0.7 \\
& Patches & 19.63 & 16.26 & 0.9 \\
\hline
\end{tabular}

Table 1. The work to fracture $W$, the apparent stiffness $E$ and the strength $F_{\mathrm{m}}$ of the different investigated structures in this study

and distribution of sticky sites. The results show that the work to fracture is mostly dependent on the number of SBs present in the system and does not dramatically change when the distribution of sticky sites is changed. For $\rho_{\mathrm{s}}=0 \cdot 08$, the work to fracture is identical within error bars for the ordered and random arrangement, while for $\rho_{\mathrm{s}}=0 \cdot 24$, it moderately increases from $W=19.63 \mathrm{eV}$ in the patches configuration to $23.51 \mathrm{eV}$ for the random arrangement, which is a change of $20 \%$ only. Thus, it is the number of SBs that has to be broken and not their distribution that mainly determines the toughness of a material. For nonreversible bonds, it is even expected that $W$ is constant because for the two extreme configurations of all bonds loaded in series or in parallel, the product of elongation and strength is constant. It is the reversibility of the SBs allowing bond reformation after rupture that is responsible for the slight increase in $W$. This situation is reversed when the behaviour of the apparent stiffness is investigated. The apparent stiffness is a measure of how much force is needed to elongate the chain. However, in contrast to the elastic modulus that is determined by the reversible elastic behaviour of a material, the apparent stiffness is a result of SB rupture, that is, plastic deformation. Nevertheless, the reversibility of the SBs allows for molecular repair and recovery of the initial mechanical parameters. The apparent stiffness for the two investigated random configurations is the same, although the number of SBs changes by a factor of 3 for the two investigated densities. Otherwise, when the number of SBs is kept constant and only their distribution is changed, the apparent stiffness changes from $E=1.84 \mathrm{meV} / \mathrm{R}^{2}$ for the random distribution to $16.26 \mathrm{meV} / \mathrm{R}^{2}$ for the patches configuration, which is almost a factor of nine. The high apparent stiffness of the patches configuration is because in contrast to the other configurations, the SBs form between sticky sites that are very close along the chain. On average, there is only one non-sticky site in between, while in the ordered configuration, this distance is at least three non-sticky sites, and all distances are possible in the random arrangement. SBs between close sticky sites rupture at large elongations; thus, the patches arrangement ensures a late but high increase in the load. When comparing the strength of the different configurations, it can be observed that for the same sticky site density, the strength of the random configuration is always the smallest, while the strength of the ordered and the patches arrangement are of similar magnitude. It is the smearing of the sacrificial force peaks over the entire length that is responsible for the reduced strength in the random distribution. The approximately twofold increase in the strength when increasing the sticky site density in the random distribution is due to the second region above $L / L_{\mathrm{C}}=0.8$ in the load-displacement curve for the random arrangement and the high sticky site density that is missing in the low-density case.

The results presented in this work suggest the following guidelines for tuning behaviour of materials based on SBs via sticky site (ligand) distribution and number. To maximize the work to fracture (toughness) of the systems, the number of SBs and their ability to reform should be maximized, regardless of the specific distribution of the SBs. For an equal number of SBs, the apparent stiffness of a material can be greatly enhanced when the SBs are distributed such that it is ensured that the force rises over a small change in length of the polymer. In the present study, this was achieved by arranging SBs in patches in particular regions of the polymer, effectively reducing the distance between sticky sites. Finally, any ordered arrangement of SBs yields an elevated strength (both ordered and patches) when compared with a purely random configuration.

\section{Conclusions}

The influence of the number and the distribution of SBs on the mechanical behaviour of single polymeric chains were investigated. It was shown that the work to fracture, related to the toughness of the material, is mainly determined by the number of SBs that have to be broken when the polymer is elongated, while the apparent stiffness and, to a lesser extent, the strength of the material is strongly dependent on the distribution of SBs in the system. The results presented have important implications for the development of new materials with tailored mechanical properties that employ sacrificial bonding - a concept that is masterly applied by nature to enhance the properties of biological materials.

\section{Acknowledgements}

S.N. and M.H. gratefully acknowledge financial support from the Austrian Science Fund (FWF) in the framework of project $\mathrm{P}$ 22983-N20. M.J.H. and P.F. acknowledge support from the Max Planck Society. 
Bioinspired, Biomimetic and Nanobiomaterials Volume 3 Issue BBN3
Influence of sacrificial bonds on the mechanical behaviour of polymer chains Nabavi, Harrington, Fratzl and Hartmann

\section{REFERENCES}

1. Fratzl, P.; Weinkamer, R. Nature's hierarchical materials. Progress in Materials Science 2007, 52, 1263-1334.

2. Smith, B. L.; Schäffer, T. E.; Viani, M., et al. Molecular mechanistic origin of the toughness of natural adhesives, fibres and composites. Nature 1999, 399, 761-763.

3. Fantner, G. E.; Oroudjev, E.; Schitter, G., et al. Sacrificial bonds and hidden length: unraveling molecular mesostructures in tough materials. Biophysical Journal 2006, 90, 1411-1418.

4. Keckes, J.; Burgert, I.; Frühmann, K., et al. Cell-wall recovery after irreversible deformation of wood. Nature Materials 2003, 2, 810-814.

5. Thompson, J. B.; Kindt, J. H.; Drake, B., et al. Bone indentation recovery time correlateswith bond reforming time. Nature 2001, 414, 773-776.

6. Fantner, G. E.; Hassenkam, T.; Kindt, J. H., et al. Sacrificial bonds and hidden length dissipate energy as mineralized fibrils separate during bone fracture. Nature Materials 2005, 4, 612-616.

7. Fantner, G. E.; Adams, J.; Turner, P., et al. Nanoscale ion mediated networks in bone: osteopontin can repeatedly dissipate large amounts of energy. Nano Letters 2007, 7, 2491-2498.

8. Becker, N.; Oroudjev, E.; Mutz, S., et al. Molecular nanosprings in spider capture-silk threads. Nature Materials 2003, 2(4), 278-283.

9. Harrington, M. J.; Wasko, S. S.; Masic, A., et al. Pseudoelastic behaviour of a natural material is achieved via reversible changes in protein backbone conformation. Journal of the Royal Society Interface 2012;9:2911-2922.

10. Harrington, M. J.; Gupta, H. S.; Fratzl, P.; Waite, J. H. Collagen insulated from tensile damage by domains that unfold reversibly: in situ X-ray investigation of mechanical yield and damage repair in the mussel byssus. Journal of Structural Biology 2009, 167, 47-54.

11. Krauss, S.; Metzger, T. H.; Fratzl, P.; Harrington, M. J. Self-repair of a biological fiber guided by an ordered elastic framework. Biomacromolecules 2013, 14, 1520-1528.

12. Hwang, D. S.; Zeng, H.; Masic, A., et al. Protein- and metaldependent interactions of a prominent protein in mussel adhesive plaques. Journal of Biological Chemistry 2010, 285, 25850-25858.

13. Qin, Z.; Buehler, M. J. Impact tolerance in mussel thread networks by heterogeneous material distribution. Nature Communications 2013, 4, 2187.

14. Keten, S.; Xu, Z.; Ihle, B.; Buehler, M. J. Nanoconfinement controls stiffness, strength and mechanical toughness of $\beta$-sheet crystals in silk. Nature Materials 2010, 9, 359-367.

15. Holten-Andersen, N.; Zhao, H.; Waite, J. H. Stiff coatings on compliant biofibers: the cuticle of Mytilus californianus byssal threads. Biochemistry 2009, 48, 2752-2759.
16. Holten-Andersen, N.; Fantner, G. E.; Hohlbauch, S.; Waite, J. H.; Zok, F. W. Protective coatings on extensible biofibres. Nature Materials 2007, 6, 669-672.

17. Lee, B. P.; Messersmith, P. B.; Israelachvili, J. N.; Waite, J. H. Mussel-inspired adhesives and coatings. Annual Review of Materials Research 2011, 41, 99-132.

18. Harrington, M. J.; Masic, A.; Holten-Andersen, N.; Waite, J. H.; Fratzl, P. Iron-clad fibers: a metal-based biological strategy for hard flexible coatings. Science 2010, 328, 216-220.

19. Barnes, D. S.; Pettit, L. D. Stereoselectivity in enthalpy changes accompanying the formation of metal complexes of histidine and other amino-acids. Journal of Inorganic and Nuclear Chemistry 1971, 33, 2177-2184.

20. Lee, H.; Scherer, N. F.; Messersmith, P. B. Single-molecule mechanics of mussel adhesion. Proceedings of the National Academy of Sciences of the United States of America 2006, 103, 12999-13003.

21. Mehdizadeh, M.; Weng, H.; Gyawali, D.; Tang, L.; Yang, J. Injectable citrate-based mussel-inspired tissue bioadhesives with high wet strength for sutureless wound closure. Biomaterials 2012;33:7972-7983.

22. Kastrup, C. J.; Nahrendorf, M.; Figueiredo, J. L., et al. Painting blood vessels and atherosclerotic plaques with an adhesive drug depot. Proceedings of the National Academy of Sciences of the United States of America 2012, 109, 21444-21449.

23. Haque, M. A.; Kurokawa, T.; Gong, J. P. Super tough double network hydrogels and their application as biomaterials. Polymer 2012, 53, 1805-1822.

24. Holten-Andersen, N.; Harrington, M. J.; Birkedal, H., et al. $\mathrm{pH}$-induced metal-ligand cross-links inspired by mussel yield self-healing polymer networks with near-covalent elastic moduli. Proceedings of the National Academy of Sciences of the United States of America 2011, 108, 2651-2655.

25. Fullenkamp, D. E.; He, L.; Barrett, D. G.; Burghardt, W. R.; Messersmith, P. B. Mussel-inspired histidine-based transient network metal coordination hydrogels. Macromolecules 2013 , 46(3), 1167-1174.

26. Barrett, D. G.; Fullenkamp, D. E.; He, L., et al. pH-based regulation of hydrogel mechanical properties through musselinspired chemistry and processing. Advanced Functional Materials 2013, 23, 1111-1119.

27. Holten-Andersen, N.; Jaishankar, A.; Harrington, M. J., et al. Metal-coordination: using one of nature's tricks to control soft material mechanics. Journal of Materials Chemistry $B$ 2014, 2, 2467-2472.

28. Xu, Z. Mechanics of metal-catecholate complexes: the roles of coordination state and metal types. Science Reports 2013, 3, 2914.

29. Lieou, C. K. C.; Elbanna, A. E.; Carlson, J. M. Sacrificial bonds and hidden length in biomaterials: a kinetic constitutive 
Bioinspired, Biomimetic and Nanobiomaterials Volume 3 Issue BBN3
Influence of sacrificial bonds on the mechanical behaviour of polymer chains Nabavi, Harrington, Fratzl and Hartmann description of strength and toughness in bone. Physical Review E 2013, 88, 012703.

30. Elbanna, A. E.; Carlson, J. M. Dynamics of polymer molecules with sacrificial bond and hidden length systems: towards a physically-based mesoscopic constitutive law. PLoS ONE 2013, 8, e56118.

31. Nabavi, S. S.; Harrington, M. J.; Paris, O.; Fratzl, P.; Hartmann, M. A. The role of topology and thermal backbone fluctuations on sacrificial bond efficacy in mechanical metalloproteins. New Journal of Physics 2014, 16, 013003.

32. Hartmann, M. A.; Fratzl, P. Sacrificial ionic bonds need to be randomly distributed to provide shear deformability. Nano Letters 2009, 9, 3603-3607.

33. Harrington, M. J.; Waite, J. H. Holdfast heroics: comparing the molecular and mechanical properties of Mytilus californianus byssal threads. Journal of Experimental Biology 2007, 210, 4307-4318.

34. Knecht, S.; Ricklin, D.; Eberle, A. N.; Ernst, B. Oligohis-tags: mechanisms of binding to Ni2+-NTA surfaces. Journal of Molecular Recognition 2009, 22, 270-279.
35. Cao, Y.; Yoo, T.; Li, H. Single molecule force spectroscopy reveals engineered metal chelation is a general approach to enhance mechanical stability of proteins. Proceedings of the National Academy of Sciences of the United States of America 2008, 105, 11152-11157.

36. Manca, F.; Giordano, S.; Palla, P. L., et al. Elasticity of flexible and semiflexible polymers with extensible bonds in the Gibbs and Helmholtz ensembles. Journal of Chemical Physics 2012, 136, 154906.

37. Landau, D.; Binder, K. A Guide to Monte Carlo Simulations in Statistical Physics. Cambridge, UK: Cambridge University Press, 2009.

38. Marko, J. F.; Siggia, E. D. Stretching DNA. Macromolecules 1995, 28, 8759-8770.

39. Ortiz, C.; Hadziioannou, G. Entropic elasticity of single polymer chains of poly(methacrylic acid) measured by atomic force microscopy. Macromolecules 1999, 32, 780-787.

40. Gutsmann, T.; Hassenkam, T.; Cutroni, J. A.; Hansma, P. K. Sacrificial bonds in polymer brushes from rat tail tendon functioning as nanoscale velcro. Biophysical Journal 2005, $89,536-542$.

\section{WHAT DO YOU THINK?}

To discuss this paper, please email up to 500 words to the managing editor at bbn@icepublishing.com

Your contribution will be forwarded to the author(s) for a reply and, if considered appropriate by the editor-inchief, will be published as a discussion in a future issue of the journal.

ICE Science journals rely entirely on contributions sent in by professionals, academics and students coming from the field of materials science and engineering. Articles should be within 5000-7000 words long (short communications and opinion articles should be within 2000 words long), with adequate illustrations and references. To access our author guidelines and how to submit your paper, please refer to the journal website at www.icevirtuallibrary.com/bbn 